\title{
Nosotras sufrimos, vosotras sufrís y ellas sufren más violencias: las usuarias de los Centros Mujer de la Comunidad Valenciana
}

\author{
Gabriela Moriana Mateo \\ Departamento de Trabajo Social y Servicios Sociales, Universitat de València \\ gabriela.moriana@uv.es
}

\begin{abstract}
Artikulu honen xedea Valentziako Erkidegoko Emakumeen Zentroetan artatutako emakumeek dituzten arazoak, ezaugarri soziodemografikoak eta artatutako emakumeen zenbatekoa aztertzea da. Horretarako, zentro horietan azken hamabi urtetako memorien datu estatistikoak aztertu dira. Emaitzei dagokienez, erabiltzaileen proportzio handienak bikotekideen edo bikotekide ohien indarkeria jasan duela esan behar da -fisikoa zein psikikoa, hurrenez hurren, psikikoa eta, askoz ere proportzio txikiagoan, sexuala-. Migratzaileen gehiegizko adierazpideak ageri arren, gehienak espainiarrak dira eta seme-alaben ardura eta dirusarrera apalagoak dituzte, hau da, lanbide arteko gutxieneko soldatatik azpiko ordainsariak jasotzen dituzte edo mendekotasun ekonomikoa dute; kasu batzuetan emakumeak babesteko zentroetan instituzionalizatu izan dira. Azken batean, lan honen ondorio nagusietako bat da pobreziak eta gizartebazterketak baldintzatuta, ezin dutela generoindarkeriatik ihes egin.
\end{abstract}

\section{GAKO-HITZAK:}

Emakumeen aurkako indarkeria, Centro Mujer, pobrezia, gizarte-bazterketa, babes-zentroak.
El objetivo de este artículo es analizar la problemática, características sociodemográficas y número de mujeres atendidas en los Centros Mujer de la Comunidad Valenciana. Para ello se han analizado los datos estadísticos de las memorias de los mencionados centros durante los últimos doce años. Respecto a los resultados, cabe señalar que la mayor proporción de usuarias ha sufrido violencia - tanto física como psíquica, seguida de psíquica y, en mucha menor proporción, sexual- por parte de sus parejas o exparejas. Aunque las migrantes están sobrerrepresentadas, mayoritariamente se trata de españolas, tienen hijas o hijos a cargo e ingresos económicos inferiores al salario mínimo interprofesional o son dependientes económicamente, por lo que en algunos casos tienen que ser institucionalizadas en los centros de protección de mujeres. Así, una de las principales conclusiones de este trabajo es que la pobreza y la exclusión social impiden a las mujeres escapar de la violencia de género.

\section{Palabras Clave:}

Violencia contra las mujeres, Centro Mujer, pobreza, exclusión social, centros de protección. 


\section{Introducción}

El objetivo de este artículo es analizar la problemática y características sociodemográficas de las mujeres atendidas en los Centros Mujer 24 Horas (en adelante $\mathrm{CM} 24 \mathrm{H}$ ) y Centros Mujer (en adelante CM) de la Comunidad Valenciana. Se trata de un recurso público de servicios sociales específicos, aunque de gestión privada, cuya finalidad es atender a las mujeres que sufren malos tratos físicos, psíquicos y sexuales (agresiones sexuales, abusos sexuales y acoso sexual en el ámbito laboral). Asimismo, se pretende analizar la evolución del número de mujeres atendidas, llamadas recibidas y usuarias derivadas de los $\mathrm{CM} 24 \mathrm{H}$ y $\mathrm{CM}$ a los centros de protección, como indicadores relacionados con el objetivo "La Atención y recuperación integral de las mujeres víctimas de violencia de género y a sus hijos e hijas", del Pacte Valencià contra la Violència de Gènere i Masclista (2017).

Los $\mathrm{CM} 24 \mathrm{H}$ y los CM realizan atención directa y telefónica en el ámbito social, psicológico y jurídico. En el servicio de atención directa informan sobre los derechos y recursos existentes para las mujeres que sufren violencia, proporcionan ayuda profesional para paliar y/o superar las consecuencias del maltrato a través de la intervención psicosocial individual y grupal, y acompañan y derivan a distintos recursos judiciales, sanitarios, formativos, laborales o de servicios sociales generales y específicos, como los centros residenciales, llamados centros de protección, casas de acogida o refugios.

Desde 1996, los CM24H han prestado su servicio en las tres provincias de la Comunidad Valenciana: Alicante, Castellón y Valencia, las 24 horas del día, los 365 días del año. A partir de 2008 se puso en funcionamiento el Centro Mujer de Denia (con horario de lunes a viernes de 08,00 a 22,00 horas); a ellos se han sumado en 2018 el CM de Torrevieja, con el mismo horario, y en 2019 los CM de Segorbe, Sant Mateu, Elda y Yátova (con horario de lunes a sábado de 09,00 a 21,00 horas).

Los centros de protección son recursos residenciales destinados a acoger a las mujeres y sus hijas o hijos durante un periodo de tiempo determinado. Siguiendo la clasificación de la Administración Pública autonómica, existen dos tipos de redes de centros de servicios sociales residenciales específicos: por una parte, para las mujeres víctimas de violencia de género y, por otra, para mujeres en riesgo de exclusión social.

Así, de acuerdo con la Ley $7 / 2012$, de 23 de noviembre, integral contra la violencia sobre la mujer en el ámbito de la Comunitat Valenciana, los centros de servicios sociales residenciales especializados para las mujeres víctimas de violencia de género se clasifican en: centros de emergencias, cuyo tiempo de estancia debe ser corto, no superior a 15-30 días, salvo excepciones muy concretas; centros de recuperación integral, donde se proporciona un tratamiento profesional integral y se facilitan los medios adecuados que permitan a las mujeres una vida independiente, en los que la duración de la estancia es de seis meses, prorrogable por un periodo similar, según la situación sociofamiliar y emocional de las mujeres acogidas y viviendas tuteladas, que proporcionan también un acogimiento de carácter temporal, con el objetivo de que las mujeres se puedan integrar con autonomía suficiente en su entorno, la duración de la estancia es de, aproximadamente, un año.

Por su parte, los centros de servicios sociales específicos para las mujeres en situación de riesgo o exclusión social se configuran como un servicio social especializado y de carácter asistencial, de protección y de promoción, cuya finalidad es acoger a mujeres solas o acompañadas de sus hijas o hijos menores y con el objeto de prestarles una atención integral, facilitándoles los medios básicos que les ayuden a su reintegración social.

Siguiendo la web del Institut de les Dones de la Generalitat Valenciana ${ }^{1}$, el acceso de las mujeres a los centros de violencia es siempre mediante la valoración de los profesionales de la red de Centros Mujer 24 Horas, que formalizan sus propuestas a la Dirección General de Familia y Mujer y a las direcciones territoriales correspondientes. El ingreso de las mujeres a los centros de exclusión social se realiza a través de las unidades de la mujer de las direcciones territoriales de la Conselleria de Alicante, Castellón y Valencia, a propuesta del personal técnico de servicios sociales. Sin embargo, como se puede comprobar en las memorias de los $\mathrm{CM} 24 \mathrm{H}$ y CM, también desde ellos se han realizado ingresos en los centros residenciales destinados a la exclusión social.

Finalmente, los $\mathrm{CM} 24 \mathrm{H}$ prestan atención telefónica, tanto en el teléfono autonómico (900580888), como en el servicio de información y asesoramiento jurídico en materia de violencia de género (016), que le fue derivado desde la Administración central en el año 2010. La atención telefónica se realiza por un equipo multidisciplinar las 24 horas del día los 365 días del año, garantizando el anonimato de este medio.

Es necesario señalar que tanto los $\mathrm{CM}_{2} 4 \mathrm{H}$ y CM como prácticamente todos los centros residenciales de la Comunidad Valenciana son de gestión privada, algunos de ellos de las mismas organizaciones religiosas que colaboraron con el régimen franquista para educar a las mujeres en la moral cristiana. Y, aunque el Pacte Valencia contra la Violencia de Gènere i Masclista (2017) se comprometió a iniciar el proceso de reversión a la titularidad pública de todos los recursos asistenciales y de intervención para víctimas, no se ha hecho nada al respecto, y ello serviría para repensar la anacrónica institucionalización de las mujeres.

${ }^{1}$ http://www.inclusio.gva.es/va/web/mujer. 


\section{Marco conceptual y metodológico}

Con el objetivo de partir de un marco común de interpretación y análisis, se van a exponer las principales definiciones normativas de violencia contra las mujeres. Así, a nivel internacional, la Declaración sobre la Eliminación de la Violencia contra la Mujer de la Organización de las Naciones Unidas (ONU, 1994), entiende por violencia contra las mujeres todo acto de violencia basado en la pertenencia al sexo femenino que tenga o pueda tener como resultado un daño o sufrimiento físico, sexual o psicológico para las mujeres, así como las amenazas de tales actos, la coacción o la privación arbitraria de la libertad, tanto si se producen en la vida pública como en la vida privada.

Esta declaración, aunque sin limitarse a ellos, abarca los siguientes tipos de violencia contra las mujeres:

- La violencia física, sexual y psicológica que se produzca en la familia, incluidos los malos tratos, el abuso sexual de las niñas en el hogar, la violencia relacionada con la dote, la violación por el marido, la mutilación genital femenina y otras prácticas tradicionales nocivas para las mujeres, los actos de violencia perpetrados por otros miembros de la familia y la violencia relacionada con la explotación.

- La violencia física, sexual y psicológica perpetrada dentro de la comunidad en general, que incluye la violación, el abuso sexual, el acoso y la intimidación sexuales en el trabajo, en instituciones educacionales y en otros lugares, la trata de mujeres y la prostitución forzada.

- La violencia física, sexual y psicológica perpetrada o tolerada por el Estado, donde quiera que ocurra.

A los efectos del Convenio de Estambul, por violencia contra las mujeres se deberá entender una violación de los derechos humanos y una forma de discriminación contra las mujeres, y designará todos los actos de violencia basados en el género que implican o pueden implicar para las mujeres daños o sufrimientos de naturaleza física, sexual, psicológica o económica, incluidas las amenazas de realizar dichos actos, la coacción o la privación arbitraria de libertad en la vida pública o privada. Asimismo, por violencia contra las mujeres por razones de género se entenderá toda violencia contra las mujeres por el hecho de serlo o que afecte a las mujeres de manera desproporcionada.

Por su parte, la Ley Orgánica, del 28 de diciembre, de Medidas de Protección Integral contra la Violencia de Género (2004), aunque de enunciado amplio, porque dice tener por objeto actuar contra la violencia que, como manifestación de la discriminación, la situación de desigualdad y las relaciones de poder de los hombres sobre las mujeres, ciñe la definición de violencia de género a: "la que se ejerce contra las mujeres por parte de quienes sean o hayan sido sus cónyuges o de quienes estén o hayan estado ligados a ellas por relaciones similares de afectividad, aun sin convivencia”. Así, la violencia de género a que se refiere esta ley comprende todo acto de violencia física y psicológica, incluida las agresiones a la libertad sexual, las amenazas, las coacciones o la privación arbitraria de libertad.

Finalmente, la Ley Integral contra la Violencia sobre la Mujer en el ámbito de la Comunitat Valenciana (2012), inspirada en el Convenio de Estambul, considera, en particular y sin carácter excluyente, que la violencia sobre la mujer se enmarca dentro de las siguientes manifestaciones: violencia física, psíquica, sexual, económica, mutilación genital femenina u otras prácticas tradicionales y/o culturales nocivas o perjudiciales para las mujeres y niñas y, finalmente, la trata de mujeres y niñas, en cualquier ámbito social. Así, hay que destacar que dicha ley hace referencia la violencia económica, que comprende toda aquella limitación, privación no justificada legalmente o discriminación en la disposición de sus bienes, recursos patrimoniales o derechos económicos, en el ámbito de convivencia de la pareja o en los casos de ruptura de la relación.

La metodología utilizada para realizar esta investigación ha sido de índole cuantitativa. Se han recopilado las memorias anuales de los CM24H y CM entre los años 2007 (que aporta alguna información del año 2006) y 2018, que la Administración autonómica ha hecho públicas en su web y se han analizado los datos estadísticos. Este periodo temporal permite conocer la cantidad de usuarias atendidas por primera vez tanto en el servicio de atención directa como telefónica, como la problemática, características sociodemográficas y su evolución.

\section{Resultados}

\subsection{Servicio de atención directa}

\subsubsection{Usuarias y problemática}

En primer lugar, en los últimos doce años, es decir, entre 2006 y 2018 , han acudido al servicio de atención directa de los $\mathrm{CM} 24 \mathrm{H}$ y $\mathrm{CM}$ de la Comunidad Valenciana, por primera vez un total de $\mathbf{2 1 . 5 2 7}$ mujeres, lo que supone una media anual de 2.159, con una tendencia al aumento en los últimos años respecto a los primeros (a esto hay que sumar una media anual de más de $\mathbf{2 . 0 0 0}$ mujeres a las que siguen atendiendo o representan casos retomadas de años anteriores).

En segundo lugar, y en cuanto a los tipos de violencia que sufren las mujeres que acuden al servicio, cabe señalar que los $\mathrm{CM} 24 \mathrm{H}$ y los $\mathrm{CM}$ utilizan una tipología que no contempla la posibilidad de violencia física solamente. Este criterio es defendido por distintas/os autoras/es, que consideran que las mujeres maltratadas físicamente también lo son psíquicamente, pues todas las personas nos sentimos 
humilladas si nos maltratan (Dutton y Golant, 1995, citados en Fontanil et al., 2004). Por ello, clasifican los malos tratos, por una parte, en físicos y psíquicos, por otra, en psíquicos y, finalmente, en sexuales. Así, entre los años 2007 y 2018, el principal tipo de maltrato que dicen haber sufrido tanto las usuarias mayores como menores de edad es la violencia física y psíquica, seguida de la psíquica y, en mucha menor proporción, sexual (Tabla 1).

Tabla 1. Tipos de malos tratos sufridos por las mujeres que acuden por primera vez a los centros de mujer entre los años 2007-2018

\begin{tabular}{|l|c|c|c|c|c|c|}
\hline \multirow{2}{*}{ Año } & \multicolumn{2}{|c|}{$\begin{array}{c}\text { Físicos } \mathbf{y} \\
\text { psíquicos }\end{array}$} & \multicolumn{2}{c|}{ Psíquicos } & \multicolumn{2}{c|}{ Sexuales } \\
\cline { 2 - 7 } & TOTAL & $\%$ & TOTAL & $\%$ & TOTAL & $\%$ \\
\hline 2007 & 1.262 & 66,0 & 548 & 28,7 & 101 & 5,3 \\
\hline 2008 & 1.527 & 67,8 & 632 & 28,2 & 89 & 3,9 \\
\hline 2009 & 1.379 & 64,9 & 641 & 30,2 & 105 & 4,9 \\
\hline 2010 & 1.423 & 65,8 & 652 & 30,1 & 88 & 4,0 \\
\hline 2011 & 1.474 & 68,2 & 608 & 28,1 & 79 & 3,7 \\
\hline 2012 & 1.350 & 67,2 & 595 & 29,6 & 63 & 3,1 \\
\hline 2013 & 1.393 & 65,9 & 641 & 30,3 & 80 & 3,8 \\
\hline 2014 & 1.480 & 67,7 & 630 & 28,8 & 77 & 3,5 \\
\hline 2015 & 1.468 & 68,0 & 618 & 28,6 & 72 & 3,4 \\
\hline 2016 & 1.371 & 66,7 & 595 & 29,0 & 89 & 4,3 \\
\hline 2017 & 1.463 & 67,4 & 623 & 28,7 & 85 & 3,6 \\
\hline 2018 & 1.570 & 65,6 & 669 & 27,9 & 156 & 6,5 \\
\hline
\end{tabular}

Fuente: Elaboración propia a partir de las memorias de los $\mathrm{CM} 24 \mathrm{H}$ y CM.
Respecto a la violencia sexual (que a su vez diferencia entre agresión sexual, abuso sexual y acoso sexual en el ámbito laboral), las usuarias informan haber sufrido (sin este dato en la memoria de 2008), sobre todo, agresiones sexuales, seguidas de abusos sexuales y, en menor proporción, acoso sexual en el ámbito laboral. A partir del año 2016 las memorias también informan de casos de trata de mujeres; así, entre 2016 y 2018 , han atendido por este tipo de violencia un total de 20 mujeres y en 2018 un caso de matrimonio forzoso.

En tercer lugar, respecto a la relación de las mujeres atendidas con las personas que las maltratan, las memorias de los años 2007 y 2008 solo proporcionan los datos de los principales agresores, sin especificar. A partir de 2009, proporcionan esta información por separado, por una parte, de los malos tratos físicos y psíquicos y, por otra, de los psíquicos; curiosamente, no facilitan estos datos de la violencia sexual. Así, entre 2009 y 2018 , la relación de las mujeres, mayores y menores de edad, tanto con los agresores de los malos tratos físicos y psíquicos, como solamente de los psíquicos, es sobre todo, de las parejas, seguidas de las exparejas, y, en muchísima menor medida, de algún miembro de la familia: hijo, padre, hermano, familiar y otros (Gráfico 1).

En cuarto lugar, las denuncias interpuestas por las usuarias del servicio muestran una tendencia clara de aumento entre los años 2006 y 2018. De hecho, durante los primeros años no llegaban a denunciar ni la mitad de las mujeres atendidas en el servicio y en los últimos lo hacen más del $64 \%$.

Gráfico 1. Relación de los agresores con las mujeres que acuden por primera vez a los centros de mujer entre los años 2007 y 2018 (en porcentaje)

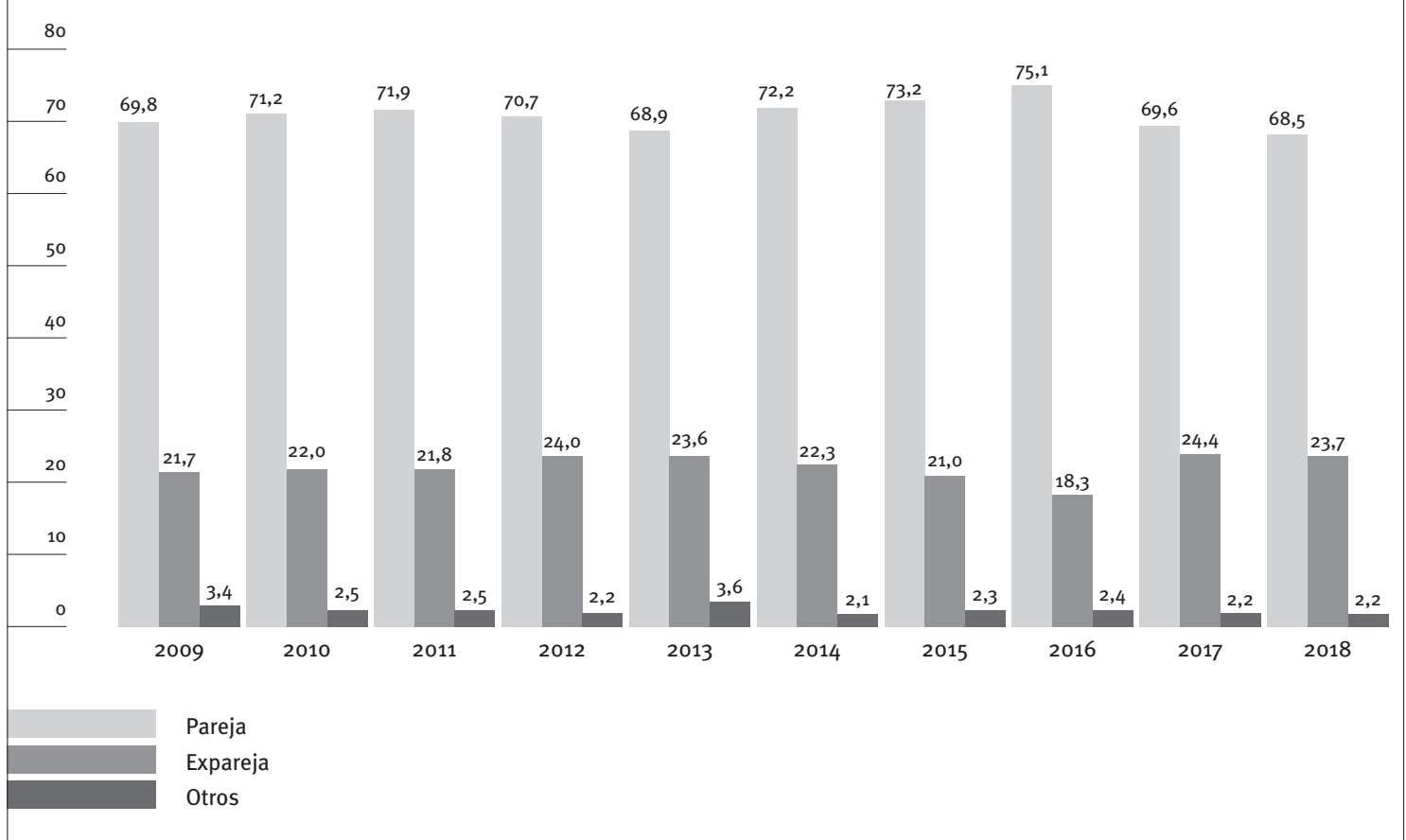

Fuente: Elaboración propia a partir de las memorias de los $\mathrm{CM} 24 \mathrm{H}$ y $\mathrm{CM}$. 
En quinto lugar, las memorias de los primeros años dejan constancia de las mujeres que acuden por primera vez que tienen o no concedida orden de protección. Sin embargo, a partir de 2016 cambian el ítem "no tienen" por "denegadas", "se desconoce" o "no procede". A pesar de ello, con los datos de las mujeres que sí tienen orden de protección sabemos que, al igual que las denuncias, la tendencia entre los años 2007 y 2018 es de claro aumento. Así, en los primeros años analizados les había sido concedida a poco más del $20 \%$ de las mujeres y en los últimos a casi la mitad (47,7\%).

\subsubsection{Características sociodemográficas de las usuarias}

En primer lugar, y en relación con la edad, entre los años 2007 y 2018 han acudido a los CM24H y CM usuarias de todas las edades. Sin embargo, el intervalo de edad más importante durante todo el periodo es el de las mujeres de entre 31 a 40 años (Tabla 2).
En segundo lugar, respecto a la procedencia de las usuarias del servicio, entre los años 2007 y 2018 la tendencia ha sido la disminución de las mujeres extranjeras, ya que en los primeros años constituían más del $30 \%$ del total y, aunque, en 2018 su número ha aumentado (28\%), los cuatro años anteriores, entre 2014 y 2017 no pasaban del $25 \%$ (Tabla 3).

Respecto al lugar concreto de procedencia de las mujeres extranjeras, se trata principalmente de mujeres europeas, a las que siguen latinoamericanas, africanas y, en último lugar, asiáticas. Entre 2009 y 2012 la mayoría de las no autóctonas eran mujeres latinoamericanas, una cifra que desciende entre los años 2012 y 2017, volviendo a aumentar en 2018. Así, son las europeas, (como señalan las memorias, especialmente, las rumanas), las que a partir de 2013 representan la mayoría de las extranjeras, aunque van en aumento las mujeres africanas (sobre todo marroquíes) y asiáticas (Tabla 4).

Tabla 2. Edad de las mujeres usuarias de los centros de mujer entre los años 2007 y 2018

\begin{tabular}{|c|c|c|c|c|c|c|c|c|c|c|c|c|c|c|c|c|}
\hline \multirow{2}{*}{ Año } & \multicolumn{2}{|c|}{ - de 18} & \multicolumn{2}{|c|}{$18-25$} & \multicolumn{2}{|c|}{$26-30$} & \multicolumn{2}{|c|}{$31-40$} & \multicolumn{2}{|c|}{ 41-50 } & \multicolumn{2}{|c|}{$51-60$} & \multicolumn{2}{|c|}{$61-70$} & \multicolumn{2}{|c|}{+ de 70} \\
\hline & $\mathbf{N}$ & $\%$ & $\mathbf{N}$ & $\%$ & $\mathbf{N}$ & $\%$ & N & $\%$ & N & $\%$ & $\mathbf{N}$ & $\%$ & $\mathbf{N}$ & $\%$ & $\mathbf{N}$ & $\%$ \\
\hline 2007 & 37 & 5,2 & 318 & 16,6 & 355 & 18,6 & 659 & 34,5 & 350 & 18,3 & 122 & 6,4 & 99 & 5,2 & 11 & 0,6 \\
\hline 2008 & 33 & 1,5 & 397 & 17,7 & 422 & 18,8 & 762 & 33,9 & 427 & 19,0 & 130 & 5,8 & 56 & 2,5 & 130 & 5,8 \\
\hline 2009 & 40 & 1,9 & 365 & 17,2 & 359 & 16.9 & 735 & 34,6 & 427 & 20,1 & 133 & 6,2 & 54 & 2,5 & 12 & 0,6 \\
\hline 2010 & 38 & 1,8 & 369 & 17,1 & 360 & 16,6 & 757 & 35,0 & 428 & 19,8 & 141 & 6,5 & 55 & 2,5 & 15 & 0,7 \\
\hline 2011 & 24 & 1,1 & 368 & 17,0 & 327 & 15,1 & 756 & 35,0 & 460 & 21,3 & 163 & 7,5 & 49 & 2,3 & 14 & 0,6 \\
\hline 2012 & 39 & 1,9 & 312 & 15,6 & 310 & 15,5 & 697 & 34,7 & 426 & 21,2 & 149 & 7,4 & 59 & 2,9 & 16 & 0,8 \\
\hline 2013 & 51 & 2,4 & 324 & 15,3 & 301 & 14,2 & 705 & 33,3 & 500 & 23,7 & 153 & 7,2 & 71 & 3,4 & 9 & 0,4 \\
\hline 2014 & 71 & 3,2 & 301 & 13,8 & 276 & 12,6 & 771 & 35,3 & 516 & 23,6 & 182 & 8,3 & 47 & 2,1 & 23 & 1,1 \\
\hline 2015 & 66 & 3,1 & 365 & 16,9 & 251 & 11,6 & 755 & 35,0 & 478 & 22,2 & 166 & 7,7 & 50 & 2,3 & 27 & 1,3 \\
\hline 2016 & 79 & 3,8 & 343 & 16,7 & 259 & 12,6 & 646 & 31,4 & 497 & 24,2 & 169 & 8,2 & 43 & 2,1 & 19 & 0,9 \\
\hline 2017 & 65 & 3,0 & 370 & 17 & 291 & 13,4 & 717 & 33,0 & 467 & 21,5 & 182 & 8,4 & 56 & 2,6 & 20 & 0,9 \\
\hline 2018 & 69 & 2,9 & 411 & 17,2 & 299 & 12,5 & 759 & 31,7 & 585 & 24,4 & 205 & 8,6 & 51 & 2,1 & 16 & 0,7 \\
\hline
\end{tabular}

Fuente: Elaboración propia a partir de las memorias de los $\mathrm{CM} 24 \mathrm{H}$ y CM.

Tabla 3. Procedencia territorial de las mujeres que acuden a los centros de mujer entre los años 207 y 2018

\begin{tabular}{|l|c|c|c|c|}
\hline \multirow{2}{*}{ Año } & \multicolumn{2}{|c|}{ Españolas } & \multicolumn{2}{|c|}{ Extranjeras } \\
\cline { 2 - 5 } & $\mathbf{N}$ & $\%$ & 627 & 32,8 \\
\hline 2007 & 1.284 & 67,2 & 789 & 35,1 \\
\hline 2008 & 1.459 & 64,9 & 658 & 31,0 \\
\hline 2009 & 1.467 & 69,0 & 712 & 32,9 \\
\hline 2010 & 1.451 & 67,1 & 636 & 29,4 \\
\hline 2011 & 1.525 & 70,6 & 571 & 28.4 \\
\hline 2012 & 1.437 & 71.6 & 550 & 26,0 \\
\hline 2013 & 1.564 & 74,0 & 508 & 23,2 \\
\hline 2014 & 1.679 & 76,8 & 541 & 25,1 \\
\hline 2015 & 1.617 & 74,9 & 533 & 25,9 \\
\hline 2016 & 1.522 & 74,1 & 550 & 25,3 \\
\hline 2017 & 1.621 & 74,7 & 679 & 28,4 \\
\hline 2018 & 1.716 & 71,6 & & \\
\hline
\end{tabular}

Fuente: Elaboración propia a partir de las memorias de los $\mathrm{CM} 24 \mathrm{H}$ y $\mathrm{CM}$. 
Tabla 4. Lugar de procedencia de las mujeres extranjeras que acuden por primera vez a los centros de mujer entre los años 2009 y 2018

\begin{tabular}{|c|c|c|c|c|c|c|c|c|}
\hline \multirow{2}{*}{ Año } & \multicolumn{2}{|c|}{ América Latina } & \multicolumn{2}{|c|}{ Europa } & \multicolumn{2}{|c|}{ África } & \multicolumn{2}{|c|}{ Asia } \\
\hline & $\mathbf{N}$ & $\%$ & N & $\%$ & $\mathbf{N}$ & $\%$ & $\mathbf{N}$ & $\%$ \\
\hline 2009 & 322 & 49,0 & 229 & 34,8 & 104 & 15,8 & 3 & 0,5 \\
\hline 2010 & 309 & 43,4 & 267 & 37,5 & 130 & 18,3 & 6 & 0,8 \\
\hline 2011 & 290 & 45,6 & 238 & 37,4 & 96 & 15,1 & 6 & 1,9 \\
\hline 2012 & 242 & 42.4 & 207 & 36,2 & 110 & 19,3 & 11 & 1,9 \\
\hline 2013 & 221 & 40,2 & 226 & 41,1 & 91 & 16,5 & 11 & 2,0 \\
\hline 2014 & 177 & 34,8 & 224 & 44,1 & 98 & 19,3 & 9 & 1,8 \\
\hline 2015 & 172 & 31,8 & 240 & 44,4 & 118 & 21,8 & 11 & 2,0 \\
\hline 2016 & 168 & 31,5 & 238 & 44,7 & 110 & 20,6 & 17 & 3,2 \\
\hline 2017 & 174 & 31,6 & 250 & 45,5 & 108 & 19,6 & 18 & 3,3 \\
\hline 2018 & 238 & 35,1 & 279 & 41,1 & 137 & 20,2 & 24 & 3,5 \\
\hline
\end{tabular}

Fuente: Elaboración propia a partir de las memorias de los $\mathrm{CM} 24 \mathrm{H}$.

En tercer lugar, entre los años 2007 y 2018, una minoría de mujeres atendidas en el servicio que oscila entre el $2,9 \%$ y el $5,7 \%$ del total anual tiene algún tipo de diversidad funcional.

En cuarto lugar, cabe señalar que la inmensa mayoría usuarias de los $\mathrm{CM} 24 \mathrm{H}$ y $\mathrm{CM}$ tienen descendencia, aunque con una tendencia a la disminución durante el periodo analizado (Gráfico 2).

Pero, además, la cantidad de hijas/os se mantiene estable: más del $40 \%$ de las mujeres tienen uno/a, del $36 \%$ al $41 \%$, dos, y entre el $18 \%$ y el $22 \%$, tres, por lo que alrededor del $60 \%$ de las usuarias del servicio tiene dos o más hijas/os.
En quinto lugar, aunque el nivel de estudios de las mujeres que han acudido al servicio por primera vez ha aumentado considerablemente entre los años 2007 y 2018 , casi la mitad no tiene estudios o solo tiene estudios primarios (Tabla 5).

En sexto lugar, y en cuanto a la procedencia de los ingresos económicos, en el periodo temporal analizado, la inmensa mayoría de las mujeres atendidas en los CM24H o CM son dependientes económicamente, porque o bien no tienen ingresos o bien cobran prestaciones y pensiones. Respecto a las mujeres que tienen trabajo remunerado, aunque la tendencia es al aumento, todavía no se ha vuelto a alcanzar el nivel anterior a la crisis económica de 2008 (Gráfico 3).

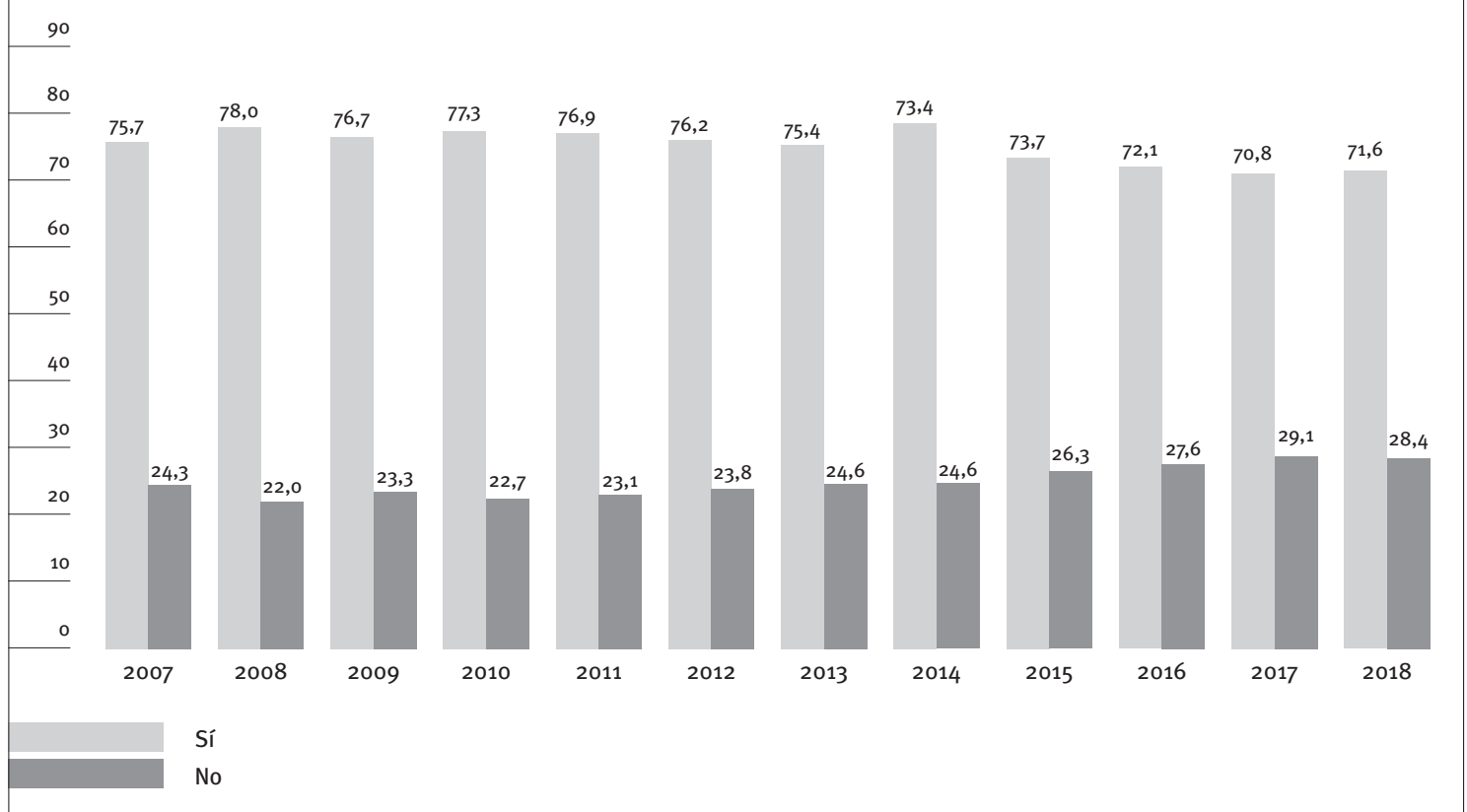

Fuente: Elaboración propia a partir de las memorias de los $\mathrm{CM} 24 \mathrm{H}$ y CM. 
Tabla 5. Nivel de estudios de las mujeres que acuden por primera vez a los CM24H entre los años 2007 y 2018

\begin{tabular}{|l|c|c|c|c|c|c|c|c|c|c|}
\hline \multirow{2}{*}{ Año } & \multicolumn{2}{|c|}{ Sin estudios } & \multicolumn{2}{|c|}{ Primaria } & \multicolumn{2}{c|}{ Secundaria } & \multicolumn{2}{c|}{ Universitaria } & \multicolumn{2}{c|}{ No consta } \\
\cline { 2 - 13 } & N & $\%$ & N & $\%$ & N & $\%$ & N & $\%$ & N & $\%$ \\
\hline 2007 & 136 & 7,1 & 842 & 44,1 & 577 & 30,2 & 254 & 13,3 & 101 & 5,3 \\
\hline $2008^{\star}$ & 134 & 6 & 890 & 39,6 & 557 & 33,7 & 265 & 11,8 & 198 & 8,8 \\
\hline 2009 & 116 & 5,4 & 794 & 37,3 & 749 & 35,4 & 268 & 12,6 & 198 & 9,3 \\
\hline 2010 & 11 & 5,1 & 815 & 37,7 & 764 & 35,2 & 262 & 12,3 & 207 & 9,6 \\
\hline 2011 & 119 & 5,5 & 872 & 40,3 & 775 & 35,8 & 279 & 12,8 & 116 & 5,4 \\
\hline 2012 & 122 & 6,1 & 794 & 39,6 & 731 & 36,3 & 249 & 12,4 & 112 & 5,6 \\
\hline 2013 & 114 & 5,4 & 820 & 38,8 & 588 & 37,3 & 279 & 13,2 & 113 & 5,3 \\
\hline 2014 & 135 & 6,2 & 807 & 36,9 & 863 & 39,4 & 275 & 12,6 & 106 & 4,8 \\
\hline 2015 & 125 & 5,8 & 766 & 36,8 & 872 & 40,4 & 323 & 14,5 & 85 & 3,9 \\
\hline 2016 & 132 & 6,4 & 723 & 35,2 & 751 & 36,5 & 364 & 17,7 & 85 & 4,1 \\
\hline 2017 & 118 & 5,4 & 697 & 32,1 & 897 & 41,3 & 367 & 16,9 & 92 & 4,2 \\
\hline 2018 & 97 & 4,1 & 753 & 31,4 & 985 & 41,1 & 436 & 18,2 & 124 & 5,2 \\
\hline
\end{tabular}

* Hay un error en las memorias, pues la suma de los absolutos no cuadra con el número de mujeres atendidas ese año. Fuente: Elaboración propia a partir de las memorias de los $\mathrm{CM}_{24} \mathrm{H}$ y $\mathrm{CM}$.

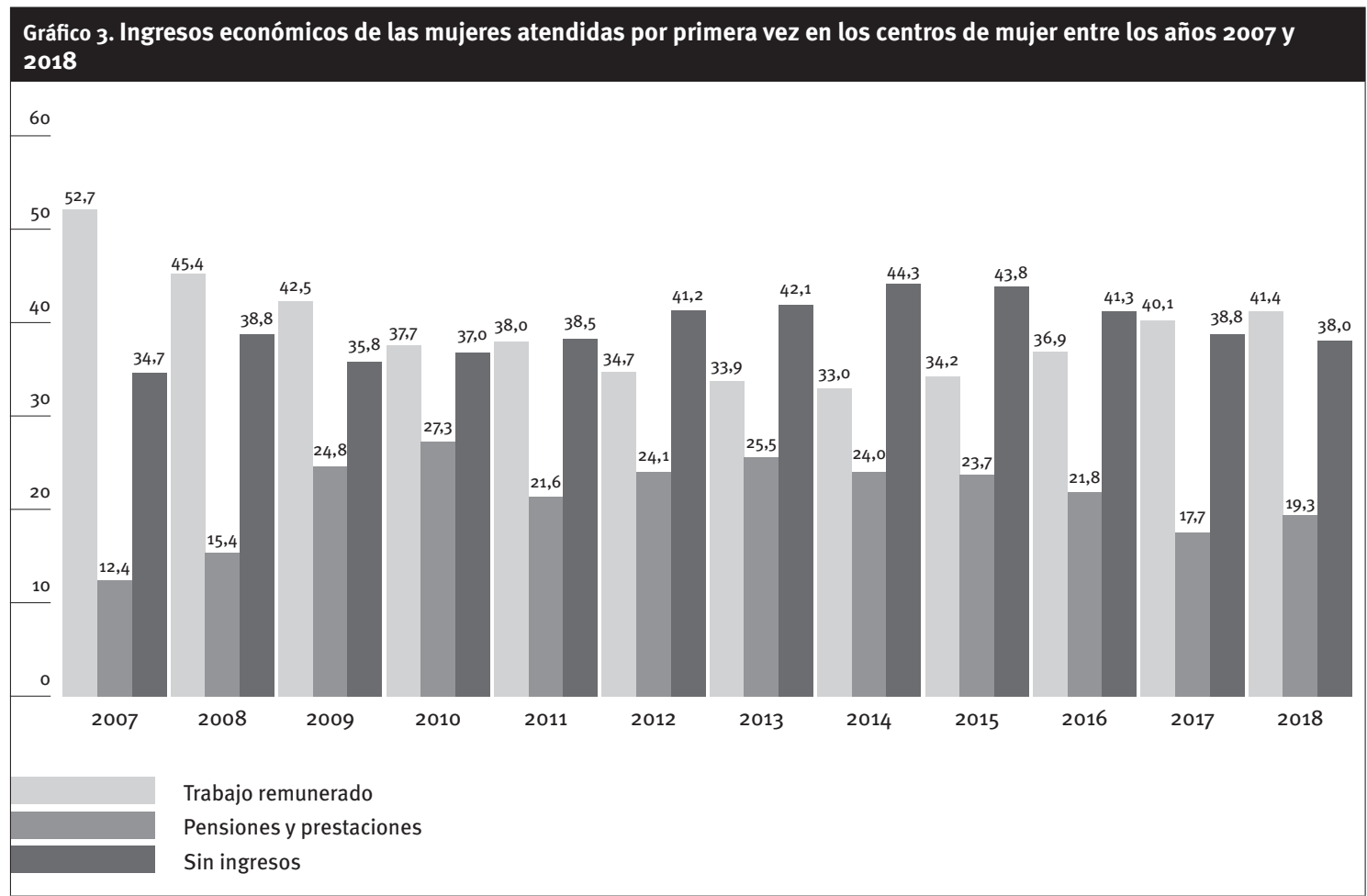

Fuente: Elaboración propia a partir de las memorias de los $\mathrm{CM} 24 \mathrm{H}$ y CM.

En séptimo lugar, entre los años 2009 y 2018 tenemos información de la cantidad de ingresos económicos. Sin embargo, las memorias algunos años contabilizan los ingresos económicos de las mujeres que los tienen por separado de las que no tienen, y otros años a las que no tienen ingresos económicos las incluyen en menos del salario mínimo interprofesional (SMI). De esta forma, restando a las mujeres que no tienen ingresos (casi el $40 \%$ ), las que sí los tienen constituyen alrededor del $60 \%$, de los que poco menos del $30 \%$ son iguales 0 inferiores al SMI y poco más del $30 \%$ superiores, por lo que el $70 \%$ de mujeres que acuden a los $\mathrm{CM} 24 \mathrm{H}$ y $\mathrm{CM}$ o bien no tienen ingresos o estos son iguales o inferiores al SMI (Tabla 6). 
Tabla 6. Cantidad de los ingresos económicos de las mujeres que acuden por primera vez a los centros de mujer entre los años 2009 y 2018

\begin{tabular}{|l|c|c|c|c|c|c|c|c|c|c|c|c|}
\hline \multirow{2}{*}{ Año } & \multicolumn{2}{|c|}{ Menos del SMI } & \multicolumn{2}{|c|}{ SMI } & \multicolumn{2}{c|}{ Entre SMI y 900 } & \multicolumn{2}{c|}{ Entre 901 y 1.200 } & \multicolumn{2}{c|}{ Más de 1.200 } & \multicolumn{2}{c|}{ No consta } \\
\cline { 2 - 16 } & $\mathbf{N}$ & $\%$ & $\mathbf{N}$ & $\%$ & $\mathbf{N}$ & $\%$ & $\mathbf{N}$ & $\%$ & $\mathbf{N}$ & $\%$ & N & $\%$ \\
\hline 2009 & 417 & 19,6 & 191 & 9 & 306 & 14,4 & 251 & 11,8 & 171 & 8,0 & 29 & 1,4 \\
\hline 2010 & 549 & 25,4 & 70 & 3,2 & 348 & 16,1 & 227 & 10,5 & 141 & 6,5 & 28 & 1,3 \\
\hline 2011 & 569 & 26,3 & 56 & 2,6 & 331 & 15,3 & 217 & 10 & 135 & 6,2 & 21 & 1 \\
\hline 2012 & 532 & 45 & 35 & 3 & 256 & 21,7 & 200 & 16.9 & 131 & 11,1 & 27 & 2,3 \\
\hline 2013 & 619 & 50,5 & 52 & 4,2 & 234 & 19,1 & 163 & 13,3 & 143 & 11,7 & 14 & 1,1 \\
\hline 2014 & 656 & 53,9 & 37 & 3 & 208 & 17,1 & 167 & 13,7 & 118 & 9,7 & 32 & 2,6 \\
\hline 2015 & 639 & 52,7 & 34 & 2,8 & 212 & 17,5 & 183 & 15,1 & 144 & 11,9 & 1 & 0,1 \\
\hline 2016 & 616 & 51 & 31 & 2,6 & 239 & 19,8 & 175 & 14,5 & 144 & 11,9 & 2 & 0,2 \\
\hline 2017 & 614 & 28,3 & 40 & 1,8 & 287 & 13,2 & 216 & 9,9 & 170 & 7,8 & 1 & 0 \\
\hline 2018 & 640 & 25,1 & 51 & 5,4 & 254 & 11,5 & 256 & 11,3 & 206 & 6,8 & 36 & 1,4 \\
\hline
\end{tabular}

Fuente: Elaboración propia a partir de las memorias de los $\mathrm{CM} 24 \mathrm{H}$ y $\mathrm{CM}$.

\section{Gráfico 4. Institucionalización de las mujeres que acuden por primera vez a los centros de mujer}

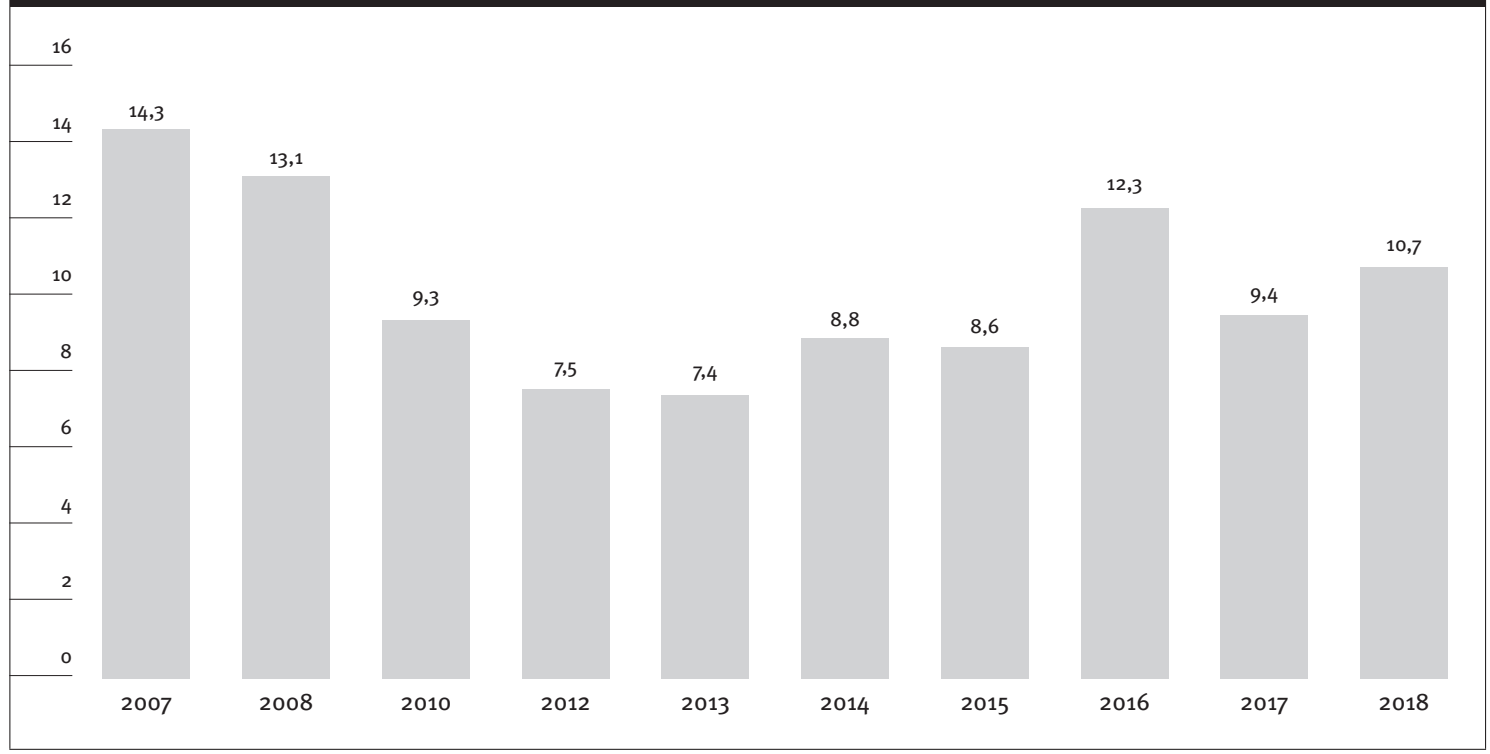

Fuente: Elaboración propia a partir de las memorias de los $\mathrm{CM}_{24} \mathrm{H}$ y CM.

Finalmente, entre un $7,4 \%$ y un $14,3 \%$ del total de las mujeres que acuden por primera vez al servicio entre los 2007 y 2018 (sin estos datos en las memorias de 2009 y 2011) han sido institucionalizadas en distintos centros residenciales de servicios sociales (Gráfico 4).

Las memorias entre los años 2016 y 2018 diferencian también entre ingresos a centros de servicios sociales específicos de violencia (entre el $82 \%$ y $87 \%$ ) y de exclusión (entre el $14,4 \%$ y el $19,4 \%$ ) y en 2018 también de menores (1,9\%) del total de institucionalizaciones.

\subsection{Servicio de atención telefónica}

En primer lugar, el servicio de atención telefónica autonómico gratuito, ha recibido entre 2006 y 2018 un total de 449.901 llamadas, una media anual de 34.607 , mensual de 2.883 y diaria de 96 , con una tendencia al aumento en los últimos años respecto a los primeros y, especialmente, en 2018.

En segundo lugar y en relación a las personas que realizan las llamadas al teléfono autonómico, entre los años 2009 y 2018, son las propias mujeres (entre el $64 \%$ y el $73 \%$ ), seguidas de las profesionales (entre el $15,5 \%$ y $19,6 \%$ ) y otras personas: familia, amistades (14,9\% y 20,6\%), con una tendencia, en el último año, a la disminución de las llamadas de las propias mujeres que sufren los malos tratos y de las profesionales, así como un aumento de las llamadas de otros.

En tercer lugar, y en cuanto a la problemática atendida en el teléfono de atención telefónica autonómico, más de la mitad de las mujeres que llaman dicen sufrir malos tratos físicos y psíquicos 
(entre un $50 \%$ y un $60 \%$ ), poco menos de la mitad psíquicos (entre un $36,6 \%$ y un $47 \%$ ) y una muy pequeña proporción sexuales (1,9\% y $3,6 \%$ ) y, de estos, sobre todo agresiones sexuales, seguidas de abuso sexual y, finalmente, acoso sexual.

Por último, con referencia al servicio telefónico de información y asesoramiento jurídico en materia de violencia de género (016) que se atiende en los $\mathrm{CM} 24 \mathrm{H}$ de la Comunidad Valenciana, entre 2010 y 2018 se han realizado una media anual de 4.948 llamadas, lo que supone una media mensual de 412 y 14 diarias.

Con relación a las personas que llaman al servicio telefónico 016, las memorias solo facilitan esa información desde el año 2010 hasta el 2016. Durante ese periodo temporal, los datos indican que son también las propias interesadas las que realizan más del $75 \%$ del total, seguidas de familiares (entre el $10 \%$ y el $13 \%$ ) y, finalmente, otros sin especificar (entre el $7,6 \%$ y el $12,4 \%$ ).

\section{Discusión}

Entre 2007 y 2018, el principal maltrato que dicen haber sufrido las mujeres (mayores y menores de edad), que han acudido o llamado al servicio, es la violencia física y psíquica, seguida de la psíquica $y$, en mucha menor proporción, la sexual. En este sentido, es necesario tener en cuenta que este tipo de malos tratos quizá esté supeditado a bajas tasas de revelación, ya que muchas mujeres no reconocen la violencia sexual ejercida por sus parejas o exparejas (Moriana, 2015b), o, como señala Ruiz (2016) en su estudio sobre adolescentes, la mayoría de las jóvenes maltratadas sufren violencia sexual camuflada por falso consentimiento.

En este sentido, la relación de las mujeres, tanto menores como mayores de edad, con los agresores de los malos tratos físicos y psíquicos, o solo psíquicos (porque, como se ha señalado, de los sexuales las memorias no proporcionan esta información), son, sobre todo, las parejas, seguida de las exparejas. Así, aunque en muchísima menor medida algunas mujeres han sufrido también otros tipos de violencia, la inmensísima mayoría de mujeres que acude a los $\mathrm{CM} 24 \mathrm{H}$ y $\mathrm{CM}$ lo hace por violencia de género de acuerdo con la ley estatal; es decir, han sido maltratadas por los hombres con los que tienen o han tenido una relación sentimental, por lo que, a la luz de estos datos, sigue siendo absolutamente pertinente y adecuada una ley específica contra la violencia hacia las mujeres por parte de sus parejas o exparejas.

El número de denuncias ha aumentado durante el periodo de tiempo analizado. Como señala Juliano (2004), este dato supone que las mujeres cada vez son más conscientes de sus derechos. Siguiendo el Boletín Estadístico Anual de la Delegación del Gobierno (2017), el número de denuncias por causa de violencia de género también ha aumentado en la Comunidad Valenciana entre los años 2007 y 2017. Asimismo, han aumentado las órdenes de protección concedidas a las usuarias de los $\mathrm{CM} 24 \mathrm{H}$ y $\mathrm{CM}$ en el periodo analizado, a diferencia de las del resto de mujeres de la Comunidad Valenciana (Delegación del Gobierno para la Violencia de Género, 2017).

Las memorias de los $\mathrm{CM} 24 \mathrm{H}$ y $\mathrm{CM}$ son una muestra clara que la violencia afecta a las mujeres de todas las edades, como ya han señalado distintas autoras y autores (Cantera, 1999; Lorente, 2001; Gil, 2007; Valls et al., 2007; Igareda y Bodelón, 2014; Moriana, 2017).

Por otra parte, la mayoría de las usuarias del servicio son españolas, aunque, en relación con su peso demográfico, las extranjeras están sobrerrepresentadas, prácticamente en la misma proporción que sus denuncias por violencia de género, tanto en la Comunidad Valenciana como en el Estado español, donde, como indican los datos estadísticos del Consejo General del Poder Judicial, son superiores al $30 \%$ del total, aunque ellas representan alrededor del $10 \%$ de la población.

La inmensa mayoría de las usuarias de los $\mathrm{CM}_{24} \mathrm{H}$ y CM tienen cargas familiares. Como se ha señalado en otros estudios (Melgar, 2009; Moriana, 2015b), las hijas/os a cargo suponen una importante barrera que impide o dificulta a las mujeres escapar de la violencia de género por diferentes motivos; por una parte, por la creencia de las madres de que existe la necesidad del padre; por otra, por la dificultad que para las mujeres solas supone compaginar el trabajo de cuidados o reproductivo con el productivo (Moriana, 2015a). Como señala el informe AROPE (2019), tener hijos/as es un importante factor de riesgo de pobreza y/o exclusión: todos los hogares en los que viven niños, niñas y adolescentes tienen tasas más altas de pobreza que aquellos donde hay personas adultas solamente. Así, dentro de ellos, subrayan especialmente la situación de los hogares monoparentales.

Alrededor de un $40 \%$ de las mujeres atendidas en los $\mathrm{CM} 24 \mathrm{H}$ y $\mathrm{CM}$ no tienen ingresos económicos y los de casi el $30 \%$ son iguales o inferiores al salario mínimo interprofesional. El estudio de la Fundación Adecco (2018), sobre las mujeres víctimas de la violencia de género señala que un $81 \%$ se encuentra desempleada o trabaja en la economía sumergida, el $65 \%$ no tiene trabajo, mientras que un $16 \%$ admite desempeñar algún tipo de ocupación pero sin contrato, en condiciones de absoluta desprotección. Por lo que subrayan que: "el desempleo y los trabajos realizados en la economía sumergida conducen a más de 8 de cada 10 mujeres víctimas a la exclusión social y a la pobreza, propiciando que su situación se perpetúe en el tiempo" (Fundación Adecco, 2018: 11).

Efectivamente, siguiendo el Informe AROPE (2019) la gran mayoría de usuarias del servicio $\mathrm{CM}_{24} \mathrm{H}$ 
y CM son pobres, ya que el umbral del riesgo de pobreza lo marcan unos ingresos de 8.871 euros anuales en hogares de una persona y de 18.629 euros para hogares de dos personas adultas y dos menroes. Como se ha señalado en distintos estudios, la carencia de recursos económicos es otra de las barreras que impide o dificulta a las mujeres que sufren violencia escapar de ella (Moriana, 2015a).

Algunas de las usuarias del servicio son derivadas o institucionalizadas en los centros de servicios sociales residenciales (tanto a los destinados a violencia como a la exclusión social) junto con su descendencia, porque, además de pobres, algunas están en procesos más o menos largos y más o menos rígidos de exclusión social. Siguiendo a Tezanos (2004: 12): “La exclusión social se define en sentido negativo, en términos de aquello de lo que se carece". Así, las mujeres ingresan en los centros residenciales por carecer, entre otros, de redes sociales y familiares que las puedan acoger, vivienda e ingresos económicos.

Como se ha señalado en algunas investigaciones (Moriana, 2014 y 2017), en las instituciones residenciales las mujeres tienen que seguir normas y horarios rígidos y convivir con otras compañeras en situaciones igual de difíciles y complicadas que ellas. Pero además, aunque la normativa dice que en centros les pueden facilitar los medios adecuados que les permitan una vida independiente para poderse integrar con autonomía suficiente en su entorno, lo que realmente hace su institucionalización es desubicarlas de su entorno solas con su descendencia, al que más pronto que tarde tendrán que volver, porque su estancia en los recursos residenciales es corta y en todos los casos finita.

Por ello, sería muchísimo más efectivo y económico (dado el importante costo de la institucionalización) protegerlas y realizar el acompañamiento desde la dependencia hasta su autonomía desde el entorno comunitario, y, si alguien tiene que salir de este, que sea el agresor. Ya es hora de centrar el control en los maltratadores, que son los que han cometido el delito, y no en las mujeres que han sufrido el maltrato. Los centros residenciales han sido y son instrumentos de control de las mujeres y de su descendencia, que desde la Edad Media hasta la actualidad perviven y evolucionan adaptándose al discurso del poder sobre la protección de las mujeres $\mathrm{y}$, como he señalado en distintas ocasiones (Moriana, 2014, 2017), donde las mujeres no quieren vivir.

Desde el enfoque interseccional, cuando las situaciones de violencia contra las mujeres se relacionan con otros ejes de desigualdad (clase, etnia o proceso migratorio, diversidad funcional), emerge una encrucijada de violencia de la que las mujeres sin recursos no pueden escapar, porque la violencia las aboca a importantes procesos de exclusión y la exclusión social, a su vez, les impide escapar de la violencia (Moriana, 2014). En este mismo sentido
Adecco (2019), señala que la salida del ciclo de la violencia es imposible cuando las mujeres no tienen independencia económica, además de que muchas se empobrecen precisamente por sufrir dicha violencia.

\section{Conclusiones}

Durante el periodo analizado, la cantidad de mujeres atendidas (nuevos casos anuales, de años anteriores y retomados) y llamadas realizadas (tanto al teléfono autonómico como al 016) en los $\mathrm{CM}_{24} \mathrm{H}$ y $\mathrm{CM}$, indican tanto el gran problema que la violencia supone para muchas mujeres, como su difícil solución.

Una de las más importantes contribuciones de este trabajo es desvelar que, aunque la violencia contra las mujeres afecta a todas las mujeres, la mayoría de las atendidas en los $\mathrm{CM} 24 \mathrm{H}$ y CM tienen cargas familiares, son migrantes, con diversidad funcional, pobres o en procesos de exclusión social. Es decir, al eje de desigualdad de género se suman otros importantes ejes de desigualdad que convierten a las mujeres es especialmente vulnerables a la violencia. Por ello, aunque la violencia afecta a todas las mujeres, no lo hace a todas de la misma manera y con la misma brutalidad, o por lo menos no para todas es tan difícil escapar de ella.

Así, es necesario que desde todos los ámbitos culturales y educativos se trabaje en la erradicación de los estereotipos, roles de género y división sexual del trabajo, que convierten a las mujeres en dependientes económicamente. Pero, además, la Administración Pública tiene que dotar a las mujeres de los recursos legales y materiales adecuados que necesiten para que puedan escapar de la violencia. Porque las mujeres que sufren violencia tienen derecho a unos ingresos económicos suficientes, y no unas prestaciones sociales como la renta activa de inserción (RAI) o la renta valenciana de inclusión, cuyo importe en ningún caso es suficiente para poder tener una vida digna e independiente. Pero también tienen derecho a residir en casas normalizadas donde ellas decidan y la Administración Pública debe concederles viviendas sociales como se han comprometido en los planes, leyes de igualdad y contra la violencia y el Pacte Valencià contra la Violencia de Gènere i Masclista (2017) y no institucionalizarlas por el hecho de no tener recursos.

De hecho, en este Pacte Valencià, la Administración Pública se compromete a: "garantizar una sociedad segura y libre de violencia de género y machista”, y a "garantizar la alternativa residencial a las mujeres víctimas de violencia de género y en situación de exclusión social para desarrollar una vida libre e independiente", señalando como necesarias, entre otras, la ampliación de la red de viviendas destinadas a las víctimas de la violencia de género y el establecimiento de convenios con promotores de vivienda para alquileres sociales, con el fin de "garantizar una vida independiente mediante 
políticas de redistribución de la riqueza, como los ingresos mínimos, y políticas de inserción sociolaboral a las mujeres víctimas de violencia de género".

Porque si la Administración Pública no les presta a las mujeres que sufren violencia de género la ayuda adecuada y suficiente, les impide escapar de ella, por lo que además de graves incumplimientos, podemos hablar de victimización secundaria o de violencia institucional, en el sentido de violencia permitida o tolerada por el Estado, de acuerdo con la Declaración de la Eliminación de la Violencia contra las Mujeres de la ONU. 
CANTERA, L. (1999): Te pego porque te quiero. La violencia en la pareja, Barcelona, Publicaciones Universitat Autònoma Barcelona.

CENTRO MUJER 24 HORAS (2009-2018): Informe anual de los Centro Mujer 24 Horas Valencia, Castellón y Alicante y Centro Mujer Denia y Torrevieja.

CENTRO MUJER 24 HORAS (2007 y 2008): Memoria anual del Centro Mujer 24 Horas.

CONSEJO DE EUROPA (2014): “Instrumento de ratificación del Convenio del Consejo de Europa sobre prevención y lucha contra la violencia contra la mujer y la violencia doméstica, hecho en Estambul el 11 de mayo de 2011", Boletín Oficial del Estado, no 137, 6 de junio, <https://www. boe.es/buscar/pdf/2014/BOE-A-2014-5947consolidado.pdf`.

CONSEJO GENERAL DEL PODER JUDICIAL: Datos estadísticos, 〈http://www.poderjudicial.es/cgpj/es/ Temas/Violencia-domestica-y-de-genero/ Actividad-del-Observatorio/Datos-estadisticos/ La-violencia-sobre-la-mujer-en-la-estadisticajudicials.

DELEGACIÓN DEL GOBIERNO PARA LA VIOLENCIA DE GÉNERO (2017): Boletín Estadístico Anual. Año 2017, <http://www.violenciagenero.igualdad. mpr.gob.es/violenciaEnCifras/boletines/ boletinAnual/docs/B_E_Anual_2017_1.pdf).

JULIANO, D. (2004): Excluidas y marginadas, Madrid, Cátedra.

FONTANIL, Y.; EZAMA, E.; FERNÁNDEZ, R. (2004): "Generación y regeneración de la violencia contra las mujeres", Cuadernos de Psiquiatría Comunitaria, volumen 4, № 1, p. 37-52.

FUNDACIÓN ADECCO (2018): 6으을 un empleo contra la violencia, 〈https://fundacionadecco.org/ wp-content/uploads/2019/03/informe-empleocontra-la-violencia.pdf〉.

GENERALITAT VALENCIANA (2012): “Ley 7/2012, de 23 de noviembre, integral contra la violencia sobre la mujer en el ámbito de la Comunitat Valenciana”, Boletín Oficial del Estado, no 297, 11 de diciembre, <https://www.boe.es/eli/es$\mathrm{vc} / \mathrm{l} / 2012 / 11 / 23 / 7 /$ con>.

- (2017): Pacte Valencià contra la Violència de Gènere $i$ Masclista, Valencia, Generalitat Valenciana, 〈https://www.sumatalpacte.com/wp-content/ uploads/2017/09/Pacte-Valencia_cast-1.pdf .

GIL, J.M. (2007): Los diferentes rostros de la violencia de género, Madrid, Dykinson.

GOBIERNO DE ESPAÑA (2004): “Ley Orgánica de Medidas de Protección Integral contra la Violencia de Género", Boletín Oficial del Estado, no 313 , 28 de enero, 〈https://www.boe.es/eli/es/ lo/2004/12/28/1/con>.

LORENTE, M. (2001): Mi marido me pega lo normal. Agresión a la mujer: realidades y mitos, Barcelona, Editorial Ares y Mares.

LLANO, J.C. (2019): El Estado de la Pobreza, 9o Informe $A R O P E$. Seguimiento del indicador de riesgo de pobreza y exclusión social en España 2008-2018. Resumen ejecutivo, Madrid, Red Europea de Lucha contra la Pobreza y la Exclusión Social, 〈https://www.eapn.es/ estadodepobreza/ARCHIVO/documentos/ Informe_AROPE_2019_Resumen_Ejecutivo.pdf〉.

MELGAR, P. (2009): “Trenquem el silenci: superación de las relaciones afectivas y sexuales abusivas por parte de las mujeres víctimas de violencia de género" [tesis doctoral], Universitat de Barcelona, 〈https://www.tdx.cat/ handle/10803/2937/>. 
MORIANA MATEO, G. (2014): “Entre la exclusión y la violencia. Las mujeres institucionalizadas en los centros de protección de mujeres de la Comunidad Valenciana" [tesis doctoral], Universitat de València, <http://gabrielamoriana.es/wp-content/ uploads/2017/02/Entre-la-exclusi\%C3\%B3n-yla-violencia.-Las-mujeres-institucionalizadasen-los-centros-de-protecci\% $\mathrm{C}_{3} \% \mathrm{~B} 3 n$-demujeres-de-la-Comunidad-Valenciana.pdf`.

- (2015a): "Barreras para escapar de la violencia de género: la mirada de las profesionales de los centros de protección de mujeres", Cuadernos de Trabajo Social, no 28-1, p. 93-102, 〈https://doi. org/10.5209/rev_CUTS.2015.v28.n1.44401>.

- (2015b): "La violencia en las historias de vida de las mujeres institucionalizadas en los centros de protección de la Comunidad Valenciana", Cuestiones de Género: de la lgualdad y la Diferencia, n- 10, p. 355-375, 〈https://doi. org/10.18002/cg.voi10.1567〉.

- (2017): "Cuando la protección es control. Las viviendas tuteladas de la Comunidad Valenciana desde el punto de vista de sus usuarias", TS Nova, $\mathrm{n}$ - 14 , p. 39-50, 〈http://cotsvalencia.com/wp-content/ uploads $/ 2018 /$ 05/TSnova_n14.pdf\#page=39>.

ORGANIZACIÓN DE NACIONES UNIDAS (1994): “Declaración de la Eliminación de violencia contra las mujeres. Asamblea General 48/104", 〈https:// www.ohchr.org/SP/Professionallnterest/ Pages/ViolenceAgainstWomen.aspx>.

RUIZ RESTREPO, C. (2016): Voces tras los datos. Una mirada cualitativa a la violencia de género en adolescentes, Instituto Andaluz de la Mujer.

TEZANOS, J.F. (2004): Tendencias en desigualdad y exclusión social, Madrid, Sistemas.

VALLS, R.; OLIVER, E.; SÁNCHEZ AROCA, M.; RUIZ EUGENIO, L.; MELGAR ALCATUD, P. (2007): “¿Violencia de género también en las universidades? Investigaciones al respecto", Revista de Investigación Educativa, vol. 25, nำ1, p. 219231, 〈https://revistas.um.es/rie/article/ view/96771/92951>. 